\title{
AMENDMENTS
}

\section{Author Correction: EMPReSS: standardized phenotype screens for functional annotation of the mouse genome}

The Eumorphia Consortium*

Correction to: Nature Genetics https://doi.org/10.1038/ng1105-1155, published online 1 November 2005.

In the version of this article initially published, members of the Eumorphia Consortium appeared in the Supplementary Information but were not included in the main article. The full list of members appears below.

\section{RAG - ANIMAGE, Lyon, France}

Marc Janier ${ }^{1}$, Mohammed Almadjub ${ }^{1}$, Olivier Beuf ${ }^{1}$, Claire Billotey ${ }^{1}$, Radu Bolbos ${ }^{1}$, Laurence Canaple ${ }^{1}$, Emmanuel Chereul ${ }^{1}$, Denis Grenier ${ }^{1}$, Bassem Hiba ${ }^{1}$, Christophe Odet ${ }^{1}$, Jean-Baptiste Langlois ${ }^{1}$, Carole Lartizien ${ }^{1}$, Luc Magnier ${ }^{1}$, Wilfried Ma ${ }^{1}$,Tarik Zouagui ${ }^{1}$

\section{CNG, Evry, France}

Mark Lathrop ${ }^{2}$, Ivo Gut ${ }^{2}$, Anne Puech ${ }^{2}$

\section{CNRS Institut de Transgenose, Orleans, France}

Yann Hérault ${ }^{3}$, Véronique Blanquet ${ }^{3}$, Veronique Brault ${ }^{3}$

ENS Lyon Laboratory of Molecular Biology of the Cell, Lyon, France

Jacques Samarut ${ }^{4}$, Denise Aubert ${ }^{5}$, Michela Plateroti ${ }^{4}$

EMBL Mouse Biology, Monterotondo, Italy

Nadia Rosenthal ${ }^{6}$, Mumna Al Banchaabouchi ${ }^{7}$, Janice Carter ${ }^{9}$, Olga Ermakova ${ }^{6}$, Karin Gale ${ }^{7}$, Jose Gonzalez ${ }^{6}$, Emerald Perlas ${ }^{6}$, Jeanette Rientjes ${ }^{6}$, Annett Spanner ${ }^{6}$, Anne-Cecile Trillat ${ }^{8}$

IBC-CNR, Monterotondo, Italy

Glauco Tocchini-Valentini ${ }^{10}$, Silvia Mandillo ${ }^{10}$, Elisabetta Golini ${ }^{10}$, Daniela Marazziti ${ }^{10}$, Giancarlo Deidda ${ }^{10}$, Nicoletta Rossi ${ }^{10}$, Rafaele Matteoni ${ }^{10}$, Marcello Raspa ${ }^{10}$, Ferdinando Scavizzi ${ }^{10}$

CNIO, Madrid, Spain

Mariano Barbacid $^{11}$, Marivi Victoria Campuzano ${ }^{11}$, Pierre Dubus ${ }^{13}$, Lucía Perez Gallego ${ }^{12}$, Carmen Guerra González ${ }^{11}$

GBF, Braunschweig, Germany

Rudi Balling ${ }^{14}$, Ursula Frischmann ${ }^{15}$, Hansjörg Hauser ${ }^{16}$, Andreas Lengeling ${ }^{17}$, Werner Müller ${ }^{15}$, Bastian Pasche ${ }^{17}$

\section{GSF (IDG \& IEG), Munich, Germany}

Martin Hrabé de Angelis ${ }^{18}$, Wolfgang Wurst ${ }^{19}$, Koichiro Abe ${ }^{18}$, Johannes Beckers ${ }^{18}$, Dirk H. Busch ${ }^{23}$, Claudia Dalke ${ }^{19}$, Valerie Gailus Durner $^{18}$, Ralf Elvert ${ }^{21}$, Tobias J. Franz ${ }^{23}$, Helmut Fuchs ${ }^{18}$, Jochen Graw ${ }^{19}$, Sabine M. Hölter ${ }^{19}$, Thilo Jakob ${ }^{25}$, Svetoslav Kalaydjiev ${ }^{23}$, Magdalena Kallnik ${ }^{19}$, Martina Klempt ${ }^{20}$, Martin Klingenspor ${ }^{21}$, Thomas Klopstock ${ }^{24}$, Christoph Lengger ${ }^{18}$, Leticia Quintanilla Martinez ${ }^{22}$, Vera Pedersen ${ }^{19}$, Oliver Puk ${ }^{19}$, Ilka Schneider ${ }^{24}$, Daniela Vogt Weisenhorn ${ }^{19}$, Sabine Wagner ${ }^{18}$, Eckhard Wolf ${ }^{20}$

Institut Clinique de la Souris, Strasbourg, France

Pierre Chambon ${ }^{26}$, Johan Auwerx ${ }^{26}$, Dalila Ali-Hadji ${ }^{27}$, Marie-France Champy ${ }^{29}$, Nelly Frossard ${ }^{31}$, Jean-Jacques Helwig ${ }^{30}$, Manuel Mark ${ }^{28}$, Laurent Monassier ${ }^{26}$, Maria Cristina Antal ${ }^{28}$, Mariette Barthelmebs ${ }^{30}$, Agnes Bloch-Zupan ${ }^{28}$, Andre Constantinesco ${ }^{26}$, Pascal Doll ${ }^{28}$, Lahcen El Fertak ${ }^{26}$, Françoise Gofflot ${ }^{28}$, Laurent Juillard ${ }^{32}$, Wojciech Krezel ${ }^{26}$, Hamid Meziane ${ }^{26}$, Abdel-Mouttalib Ouagazzal ${ }^{26}$, Françoise Pons $^{31}$, Raymond Romand ${ }^{26}$, Mohammed Selloum ${ }^{29}$, Tania Sorg ${ }^{26}$, Marius Teletin ${ }^{28}$, Laurent Vasseur ${ }^{26}$, Olivia Wendling ${ }^{28}$

\section{Institut Pasteur, Paris, France}

Philip Avner ${ }^{33}$, Sophie Chantalat ${ }^{33}$

\section{Karolinska Institute, Sweden}

Urban Lendahl ${ }^{34}$, Johannes Wilbertz ${ }^{34}$

\section{MRC Functional Genetics Unit, UK}

Kay Davies ${ }^{35}$, Peter Oliver ${ }^{35}$

MRC Harwell (MGU and MLC), UK 
Steve Brown ${ }^{36}$, Michael Cheeseman ${ }^{37}$, Emma Coghill $^{36}$, Roger $\operatorname{Cox}^{36}$, Liz Bentley ${ }^{36}$, Simon Ferguson ${ }^{36}$, Hilary Gates ${ }^{36}$, Georgios Gkoutos ${ }^{36}$, Eain Green ${ }^{36}$, John Hancock ${ }^{36}$, Tertius Hough ${ }^{36}$, Robert Johnson ${ }^{36}$, Heena Lad ${ }^{36}$, Ann-Marie Mallon ${ }^{36}$, Pat Nolan ${ }^{36}$, Andy Parker ${ }^{36}$, Julie Quarterman ${ }^{36}$, Adrian Smith ${ }^{36}$, Mandy Studley ${ }^{36}$, Aadya Shukla ${ }^{36}$, Hilda Tateossiann ${ }^{36}$, Valter Tucci ${ }^{36}$, Joseph Weekes ${ }^{36}$

MRC Human Genetics Unit, Edinburgh, UK

Ian Jackson ${ }^{38}$, Sally Cross ${ }^{38}$, Duncan Davidson ${ }^{38}$, Alan Hart ${ }^{38}$, Angus Murray ${ }^{38}$

NKI, Amsterdam, Netherlands

Anton Berns ${ }^{39}$, Marco Breuer ${ }^{40}$, Evert van Garderen ${ }^{43}$, Jos Jonkers ${ }^{41}$, Ji Ying Song ${ }^{42}$, Martin van der Valk ${ }^{42}$

\section{University of Manchester, UK}

Ludwig Neyses ${ }^{44}$, Elizabeth Cartwright ${ }^{45}$, Delvac Oceandy ${ }^{45}$, Michael Emerson ${ }^{46}$

University of Lausanne, Switzerland

Walter Wahli ${ }^{47}$, Maria Belen Delgado ${ }^{47}$, Batrice Desvergne ${ }^{47}$, Liliane Michalik ${ }^{47}$, Elodie Bedu ${ }^{47}$

University of Geneva, Switzerland

Denis Duboule ${ }^{48}$, Marie Kmita ${ }^{48}$, Jozsef Zakany ${ }^{48}$, Francois Spitz ${ }^{48}$

\section{The Wellcome Trust Sanger Institute, Cambridge, UK}

Allan Bradley ${ }^{49}$, Madhuri Warren ${ }^{49}$

${ }^{1}$ Rhone-Apes Genopole - ANIMAGE Bat. CERMEP, 59 Boulevard Pinel, 69003 Lyon, France.

${ }^{2}$ Centre Nationale de Genotypage, 2 Rue Gaston Cremieux, CP 5721, 91057 Evry CEDEX, France.

${ }^{3} \mathrm{CNRS}$ Institut de Transgenose, IEM, 3B rue de la ferollerie 45071 Orleans cedex 2, France

${ }^{4}$ Ecole Normale Superieure de Lyon, Laboratory of Molecular Biology of the Cell, 46 Allée d'Italie, 69364 Lyon Cedex 07, France.

${ }^{5}$ Ecole Normale Suprieure de Lyon, PBES, 46 Alle d'Italie, 69364 Lyon cedex 07, France.

${ }^{6}$ EMBL Mouse Biology Unit, via Ramarini 32, 00016 Monterotondo Scalo, Rome, Italy.

${ }^{7}$ EMBL Mouse Phenotyping Core, via Ramarini 32, 00016 Monterotondo Scalo, Rome, Italy.

${ }^{8}$ De Simone \& Partners, Intellectual Property Attorneys, Via VincenzoBellini 20, 00198 Rome, Italy.

${ }^{9}$ University of Cambridge, Clinical Biochemistry, Addenbrooke's Hospital, Box 232, Hills Road, Cambridge CB2 2QR, UK.

${ }^{10}$ IBC-CNR, Campus "A.Buzzati-Traverso", via Ramarini 3200016 Monterotondo Scalo, Rome, Italy.

${ }^{11}$ CNIO- Centro Nacional de Investigaciones Oncologicas, Molecular Oncology, Melchor Fernandez Almagro 3, 28029 Madrid, Spain.

${ }^{12} \mathrm{CNIO}$ - Centro Nacional de Investigaciones Oncologicas, Comparative Pathology, Melchor Fernandez Almagro, 328029 Madrid, Spain.

${ }^{13}$ University of Bordeaux, Histology and Molecular Pathology Department, University of Bordeaux 2, EA2406 146 Rue Leo Saignat 33076 Bordeaux, France.

${ }^{14}$ GBF- German Research Centre for Biotechnology, Mascheroder Weg1, 38124, Braunschweig, Germany.

${ }^{15} \mathrm{GBF}$ - German Research Centre for Biotechnology, Experimental Immunology, Mascheroder Weg1, 38124, Braunschweig, Germany.

${ }^{16}$ GBF- German Research Centre for Biotechnology, Gene Regulation and Differentiation, Mascheroder Weg1, 38124, Braunschweig, Germany.

${ }^{17}$ GBF- German Research Centre for Biotechnology, Infection Genetics, Mascheroder Weg1, 38124, Braunschweig, Germany.

${ }^{18}$ GSF- National Research Centre for the Environment and Health, Institute of Experimental Genetics, Ingolstaedter Landstrasse 1, D- 85764 Neuherberg, Germany.

${ }^{19} \mathrm{GSF}$ - National Research Centre for the Environment and Health, Institute of Developmental Genetics, Ingolstaedter Landstrasse 1, D- 85764 Neuherberg, Germany.

${ }^{20}$ Institute of Molecular Animal Breeding and Biotechnology, Gene Center, LMU Munich, Feodor-Lynen-Str. 25, 81377 Munich, Germany.

${ }^{21}$ Philipps University Marburg, Biology Department, Animal Physiology, Karl-von-Frisch Str. 8, D-35032 Marburg, Germany.

${ }^{22}$ GSF - National Research Center for Environment and Health and Health, Institute of Pathology, Ingolstaedter Landstrasse 1, D- 85764 Neuherberg, Germany.

${ }^{23}$ Institute for Medical Microbiology, Immunology and Hygiene, Technical University of Munich, Trogerstrasse 9, 81675 Munich, Germany. 
${ }^{24}$ Department of Neurology, Klinikum Großhadern, LMU Ludwig-Maximilians-University, Munich, Germany.

${ }^{25}$ Division of Environmental Dermatology and Allergy TUM/GSF, Department of Dermatology and Allergy, Technical University of Munich, Biedersteiner Strasse 29, 80802 Munich, Germany.

${ }^{26}$ ICS-Institut Clinique de la Souris, 1 Rue Laurent Fries, BP10142, 67404 Illkirch, Cedex, France.

${ }^{27}$ ICS-Institut Clinique de la Souris, Animal Facility, 1 Rue Laurent Fries, BP10142, 67404 Illkirch, Cedex, France.

${ }^{28}$ ICS-Institut Clinique de la Souris, Histopathology and Embryology, 1 Rue Laurent Fries, BP10142, 67404 Illkirch, Cedex, France.

${ }^{29}$ ICS-Institut Clinique de la Souris, Biochemistry, 1 Rue Laurent Fries, BP10142, 67404 Illkirch, Cedex, France.

${ }^{30}$ ICS-Institut Clinique de la Souris, Renovascular Pharmacology and Physiology Laboratory, U727 INSERM, Facult de M decine, 11 rue Humann, 67085 Strasbourg Cedex, France.

${ }^{31}$ ICS-Institut Clinique de la Souris, Respiratory Pharmacology and Physiology, Facult de Pharmacie, 74 route du Rhin, BP 6002467401 Illkirch Cedex, France.

${ }^{32}$ Service de Néphrologie-Hypertension artérielle, Hôpital Edouard Herriot Pavillon P, 5 place d’Arsonval, 69437 Lyon cedex 03, France. ${ }^{33}$ Instititut Pasteur, 25 rue du Docteur Roux, 75724 PARIS CEDEX 15, France.

${ }^{34}$ Karolinska Institute, Department of Cell and Molecular Biology, Von Eulers v g 3, 17177 Stockholm, Sweden.

${ }^{35}$ MRC- Medical Research Council Functional Genetics Unit, Department of Human Anatomy and Genetics, University of Oxford, South Parks Road, Oxford, OX1 3QX UK

${ }^{36}$ MRC- Medical Research Council Mammalian Genetics Unit, Harwell, Oxfordshire, OX11 0RD, UK.

${ }^{37}$ MRC- Medical Research Council Mary Lyon Centre, Harwell, Oxfordshire, OX11 0RD, UK.

${ }^{38}$ MRC- Medical Research Council Human Genetics Unit, Comparative and Developmental Genetics Department, Western General Hospital, Crewe Road, Edinburgh, EH4 2XU, UK.

${ }^{39}$ NKI- Netherlands Cancer Institute, Plesmanlaan 121, 1066 CX Amsterdam, Netherlands.

${ }^{40}$ NKI- Netherlands Cancer Institute, Animal Facility, Plesmanlaan 121, 1066 CX Amsterdam, Netherlands.

${ }^{41}$ NKI- Netherlands Cancer Institute, Department of Molecular Biology, Plesmanlaan 121, 1066 CX Amsterdam, Netherlands.

${ }^{42}$ NKI- Netherlands Cancer Institute, Department of Experimental Animal Pathology, Plesmanlaan 121, 1066 CX Amsterdam, Netherlands.

${ }^{43}$ Gezondheidsdienst voor Dieren B.V., Arnsbergstraat 7, Postbus 9, 7400 AA Deventer, Netherlands.

${ }^{44}$ University of Manchester, Manchester Heart Centre, Manchester Royal Infirmary, Oxford Road, Manchester M13 9WL, UK.

${ }^{45}$ University of Manchester, Medical school, Rm 1.302, Stopford building, University of Manchester, Oxford Road, Manchester, M13 9PT, UK.

${ }^{46}$ Imperial College, Faculty of Life Sciences, Imperial College London, Room 211, Biochemistry Building, Exhibition Road, South Kensington, London SW7 2AZ, UK.

${ }^{47}$ National Centre of Research 'Frontiers in Genetics', University of Lausanne, Center for Integrative Genomics, Le Genopode, CH-1015 Lausanne, Switzerland.

${ }^{48}$ National Centre of Research 'Frontiers in Genetics', University of Geneva, Department of Zoology and Animal Biology, Sciences III, 4031b, 32, Boulevard d’Yvoy, 1211 Geneva 4, Switzerland.

${ }^{49}$ The Wellcome Trust Sanger Institute, Team 82, Wellcome Trust Genome Campus, Hinxton, Cambridge, CB10 1SA, UK

${ }^{\star} \mathrm{A}$ list of authors and their affiliations appears online.

Published online: 15 February 2022

https://doi.org/10.1038/s41588-022-01019-w

(c) Springer Nature America, Inc. 2022 